\title{
EFFECTIVE FIELD THEORY IN NUCLEAR PHYSICS
}

\author{
Martin J. Savage ${ }^{a, b *}$ and Barry R. Holstein ${ }^{c}$ \\ ${ }^{a}$ Department of Physics, University of Washington, \\ Seattle, WA 98195. \\ b Jefferson Laboratory, 12000 Jefferson Avenue, \\ Newport News, VA 23606. \\ c Department of Physics, University of Massachusetts, \\ Amherst, MA 01003.
}

\begin{abstract}
The Electromagnetic and Hadronic physics sub-community of nuclear physics held a town hall meeting at Jefferson Lab during November 30 to December 4 of 2000 . This is is our combined contribution to the white paper that will result from this meeting.
\end{abstract}

\section{THE FUNDAMENTAL SCIENTIFIC QUESTIONS ADDRESSED}

A fundamental challenge facing the nuclear physics community is the development of a theory that describes the properties and dynamics of the strongly interacting mesons and baryons. This theory must be consistent with QCD for processes below the chiral symmetry breaking scale, a scale comparable with the nucleon mass. The importance of meeting this challenge cannot be overstated as it is vital for a theoretical description of nuclei and multi-nucleon processes, including electroweak probes. Further, it is necessary in order to make reliable predictions in conditions that are not accessible to controlled measurement, such as those that exist in supernovae, or during hadronization following the formation of a quark-gluon plasma.

Effective field theory (EFT) organizes quantum field theories according to hierarchies of physical scales [1]. The Standard Model of electroweak interactions is a beautiful ex-

*NT@UW-00-036 
ample of an EFT, which describes observables at energies below the scale of electroweak symmetry breaking. Its reliability and rigor are undisputed. An EFT is the most general description that exists consistent with all underlying symmetries and physical principles. The uncertainty associated with a calculation of any observable in an EFT can be estimated and controlled. It is sometimes the case that in a particular limit of the parameter space of the underlying theory, additional symmetries become manifest, e.g. chiral and heavy-quark symmetries in QCD. The EFT then allows one to calculate perturbatively about the symmetry limit. To describe hadronic interactions, a dual expansion in the up and down quark masses, $m_{u}$ and $m_{d}$, and in the momentum of external probes is required. This approach was pioneered by Weinberg [2] and has been successfully applied in the meson sector including both two- and three-flavors [3], and in the single nucleon sector (for a recent review see Ref. [4] $)$. This body of work, collectively known as chiral perturbation theory $(\chi \mathrm{PT})$, provides a cornerstone in our understanding of QCD and is the only rigorous way in which to encode the entire body of QCD predictions at low energy. Nevertheless, challenges remain in its development, as will be discussed below.

The last decade has also seen important progress in the extension of the hadronic EFT to systems involving more than one nucleon, that is to say, toward the construction of nuclear chiral perturbation theory $(\mathrm{N} \chi \mathrm{PT})$. The intrinsically nonperturbative nature of nuclei renders this endeavor highly nontrivial. Influential work by Weinberg [5] in the early 1990's and by many others (for a review and extensive referencing for what follows see Ref. [6]) in the late 1990's has led to remarkable progress in describing multi-nucleon systems at low energies. A few samples will be discussed below. There are, however, many unresolved issues about how best to organize and optimize the EFT expansion, in particular as regards the treatment of the pion. Resolution of these issues is essential in order to develop a systematic expansion about the chiral limit and to push the range of validity of the EFT beyond the Fermi momentum of nuclear matter.

\section{MAJOR ACHIEVEMENTS SINCE THE LAST LONG RANGE PLAN}

\section{A. Chiral Perturbation Theory}

Chiral perturbation theory was discovered by Weinberg [2] in the late 1960's and 1970's. Subsequently, it was developed in the early 1980's by Gasser and Leutwyler [3] who wrote down the most general counterterm Lagrangian for mesons at one-loop order $\left(\mathcal{O}\left(p^{4}\right)\right)$ including ten apriori unknown parameters, commonly known as the $L_{i}$ 's. By comparing the 
one-loop order predictions with experiment, empirical values for the $L_{i}$ can be obtained. Given the simplicity of the theory at one-loop order, it is quite predictive, despite the appearance of the $L_{i}$ 's. Examples are shown in Table 1, where predictions are compared with experimental determinations for quantities that receive contributions from just two of the constants - $L_{9}, L_{10}$. The table reveals at least one intriguing problem - the solid prediction of $\chi \mathrm{PT}$ for the electric polarizability of the $\pi^{+}$may be violated. However, of the three experimental results only one can be considered to be in disagreement. Clearing up this discrepancy should be a focus of future experimental work in this area.

\begin{tabular}{cccc}
\hline \hline Reaction & Quantity & Theory & Experiment \\
\hline$\pi^{+} \rightarrow e^{+} \nu_{e} \gamma$ & $h_{V}\left(m_{\pi}^{-1}\right)$ & 0.027 & $0.029 \pm 0.017$ [0]] \\
$\pi^{+} \rightarrow e^{+} \nu_{e} e^{+} e^{-}$ & $r_{V} / h_{V}$ & 2.6 & $2.3 \pm 0.6[0]$ \\
$\gamma \pi^{+} \rightarrow \gamma \pi^{+}$ & $\left(\alpha_{E}+\beta_{M}\right)\left(10^{-4} \mathrm{fm}^{3}\right)$ & 0 & $1.4 \pm 3.1[8]$ \\
& $\alpha_{E}\left(10^{-4} \mathrm{fm}^{3}\right)$ & 2.8 & $6.8 \pm 1.4[9]$ \\
& & $12 \pm 20[10]$ \\
\hline \hline
\end{tabular}

TABLE I. Predictions of $\chi \mathrm{PT}$ and data for radiative pion processes.

$\chi \mathrm{PT}$ has been extended to include baryons where it is found that in order to have a consistent power-counting it is necessary to perform a simultaneous expansion in energymomentum and in inverse powers of the nucleon mass [12]. This procedure is called heavy baryon chiral perturbation theory $(\mathrm{HB} \chi \mathrm{PT})[12]$ and has been used to address most of the problems in low energy baryonic interactions [4. As opposed to the case of the mesons discussed above, however, there are issues which are still not completely understood. The convergence of the perturbative expansion is slower than in the meson sector and in some cases chiral SU(3) loops produce large effects which must be partially canceled by the corresponding counterterms. Both these problems are under study. However, there are a large number of successful predictions with which to challenge experiment, especially in the realm of Compton scattering, both real and virtual. In many cases, calculations and empirical numbers are available for the polarizabilities and generalized polarizabilities [13], which characterize the response of a system to an applied electromagnetic field. Also, within the last few years precision experimental results for near threshold pion photoproduction and electroproduction have become available. Theoretical calculations at $\mathcal{O}\left(p^{4}\right)$ generally compare well with data but convergence may be a problem for the S-wave multipoles.

In order to make a connection between the numerical results of present day lattice- 
QCD efforts (partially-quenched and with unphysical quark masses) and nature, the particle physics community has developed an effective field theory tool [14]. This tool is known as partially-quenched- $\chi \mathrm{PT}(\mathrm{PQ} \chi \mathrm{PT})$, and is being used successfully in the particle physics lattice-QCD community.

In general, a reliable calculational framework in both the meson and single nucleon sectors exists with which to confront precise experimental data. However, important theoretical challenges remain:

i) Understanding how to deal with convergence issues, especially in the nucleon sector.

ii) Understanding how to take this reliable calculational formalism to higher energies than are presently possible.

iii) Extending existing calculations to sectors wherein present calculations are lacking, e.g. processes involving the $\eta$ and $\eta^{\prime}$.

iv) Understanding how to calculate the empirical constants from first principles, rather than dealing with them phenomenologically.

Work is underway on these issues at the present time:

i) Extending specific calculations to two-loop order [15].

ii) Attempts to marry chiral and dispersive methods to achieve unitary amplitudes which are valid to much higher energies than previously thought possible 16.

iii) Calculations involving both the $\eta$ and $\eta^{\prime}$ [17.

iv) Analytical techniques combined with lattice-QCD efforts in the particle theory community have begun to confront the empirical values of the $L_{i}$ 's with underlying theory.

v) Work which marries chiral and $1 / N_{c}$ methods [18.

\section{B. Multi-Nucleon EFT}

For very low-energy processes, involving energy and momentum much less than the pion mass, an EFT in the two-nucleon sector has been developed, $\operatorname{EFT}(\not)$, which allows for 
a perturbative calculation of processes involving two nucleons. Consider $n p \rightarrow d \gamma$ and $\nu d-$ break-up. First, the cross section, $\sigma_{n p}$, for the radiative capture $n p \rightarrow d \gamma$ plays a central role in predicting the abundance of elements from Big-Bang nucleosynthesis (BBN). For many years an error of $5 \%$ was assigned to $\sigma_{n p}$. Recently, $\sigma_{n p}$ was computed in $\operatorname{EFT}(\hbar)$, and is now described at the $\sim 1 \%$ level by a compact analytic expression. Second, cross sections for $\nu d$-break-up are required input in order to determine the flux of neutrinos from the sun with the Sudbury Neutrino Observatory (SNO). The differences among existing potential model calculations are at the $\sim 5 \%$-level, arising primarily from differing treatment of mesonexchange-currents (MEC's). Using $\operatorname{EFT}(\hbar)$, it has been shown that in order to perform a $\sim 1 \%$ calculation of this cross section, one a priori unknown coefficient, $L_{1, A}$ needs to be determined. Comparisons between the analytic EFT calculation and the numerical potential model calculations are shown in Figure 1. As it is likely to be many years before lattice-QCD
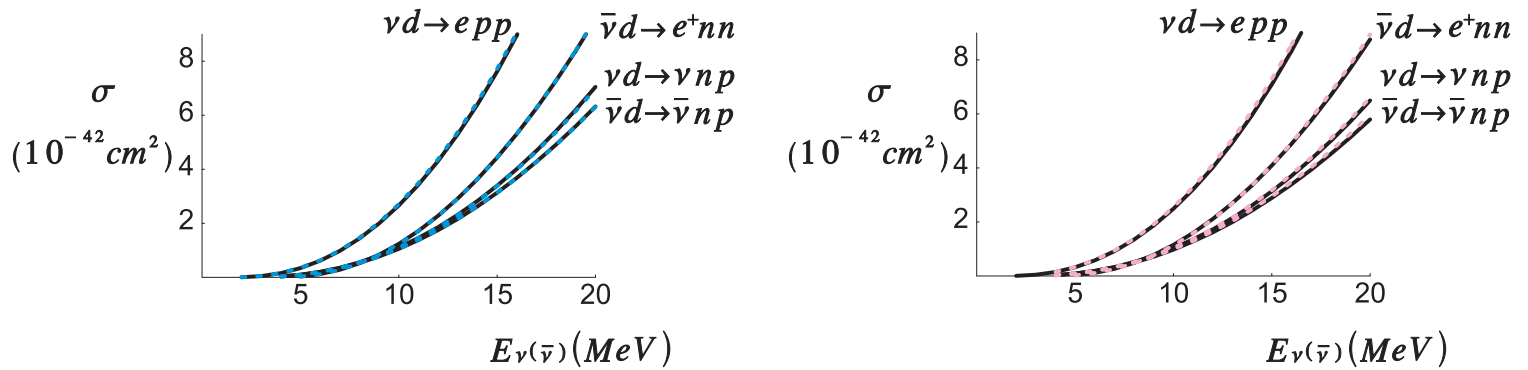

FIG. 1. Inelastic $\nu(\bar{\nu}) d$ cross sections as a function of incident $\nu(\bar{\nu})$ energy. In the left panel the solid curve is the result of the potential model calculation of Ref. [19] while the dashed curve is the $\operatorname{EFT}(\hbar)$ result with $L_{1, A}=5.6 \mathrm{fm}^{3}$ [20]. In the right panel the solid curve is the result the potential model calculation of of Ref. [21] while the dashed curve is the $\operatorname{EFT}(\hbar)$ result with $L_{1, A}=0.94 \mathrm{fm}^{3}$ 20].

can produce a value for $L_{1, A}$, an experiment to measure the cross section of $\nu_{e} d \rightarrow p p e^{-}$at the $\sim 1 \%$-level is supported. Such a measurement will allow for a $\sim 1 \%$-level prediction of the other break-up channels. In addition to these two processes, $\operatorname{EFT}(\hbar)$ has been used to make precise predictions for other two-nucleon observables, such as $\gamma d \rightarrow \gamma d$ Compton scattering that may, with precise experimental measurements at low-energies, yield reliable determinations of the polarizabilities of the neutron. Significant progress has also been made in the computation of three-body scattering cross sections and in our understanding of how multi-nucleon operators, such as the three-body force, contribute to low-energy processes. Spectacular results have been obtained in the spin- $\frac{3}{2}$ channel, where the $n d$ scattering length has been computed at next-to-next-to-leading order in perturbation theory to be $a_{3 / 2}^{\text {EFT }}=$ $6.33 \pm 0.05 \mathrm{fm}$, which is to be compared with the experimental determination of $a_{3 / 2}^{\text {expt }}=$ $6.35 \pm 0.02 \mathrm{fm}$ (subsequent second generation potential model calculations also agree with $a_{3 / 2}^{\text {expt }}$ ). The calculation of the energy dependence is similarly impressive. The Phillips line, 
relating the triton binding energy and the three-body scattering length is recovered, and has been shown to result from the freedom associated with choice of three-body force. In addition, $N d$ scattering has been studied in the p-wave and higher partial waves, producing very nice predictions that are yet to be confirmed experimentally. The techniques developed for the three-nucleon systems have been successfully applied to some observables in the area of Bose-Einstein condensation (BEC). On a more technical note, the appearance of a oneparameter limit-cycle in the renormalization group evolution of the three-body force is of current interest.

The EFT description including pions has also advanced significantly. Weinberg's original proposal to compute $N N$ potentials using the organizational principles of $\chi \mathrm{PT}$ has been widely developed. Nucleon-nucleon scattering phase shifts, $\gamma d$ Compton scattering, $\gamma d \rightarrow \pi^{0} d$ (see Figure 2 for a comparison of the threshold prediction with data), and other

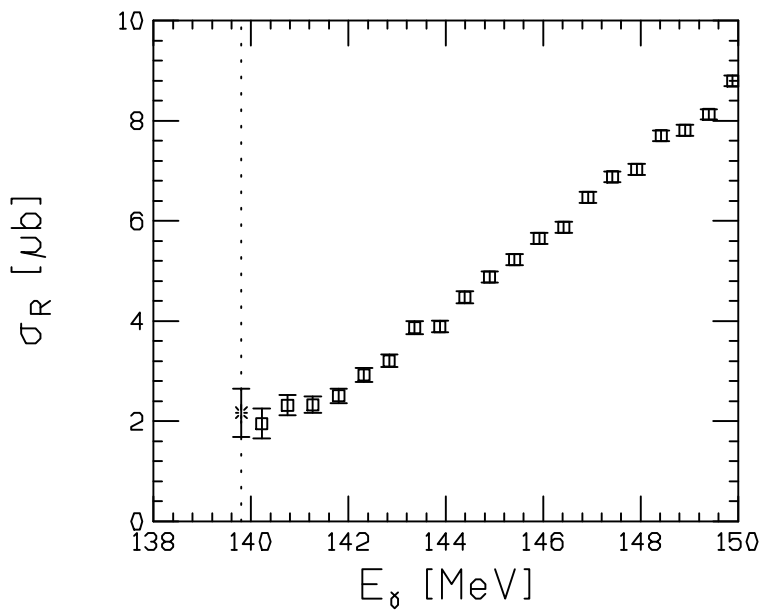

FIG. 2. The total cross section for $\gamma d \rightarrow \pi^{0} d$. Data from SAL are depicted by the boxes, while the chiral perturbation theory threshold prediction and its associated uncertainty (computed prior to the experiment) is the star and error bar on the vertical dotted line (threshold photon energy).

inelastic processes have been computed with great success using Weinberg power counting. Three-body calculations at third order in Weinberg power counting are underway, and preliminary results appear to provide insight into the well-known $A_{y}$ puzzle. Weinberg's method is intrinsically numerical and is similar in spirit to traditional nuclear physics potential theory. Unfortunately, the renormalization group scaling of operators in this EFT is complicated. Moreover, there appear to be inconsistencies in the handling of divergences proportional to the quark masses, which can potentially lead to uncontrolled errors. In contrast, KSW power counting, where pions contribute at subleading orders in the expansion, and which allows for analytic results, is found not to converge at higher orders in the spintriplet channels. In exploring these two different types of power counting a large amount 
of expertise has been acquired, and efforts are ongoing to formulate a consistent and converging power counting, that is sure to involve ingredients from both Weinberg and KSW power-counting schemes.

Work is underway to incorporate the rigor introduced by the EFT framework into the nuclear many-body problem. While this effort is still in its infancy, very encouraging

results have been obtained which suggest that these new techniques will lead to a dramatic simplification in this arena.

\section{SHORT TERM ( $<3$ YRS) AND LONG TERM ( $<10$ YRS) OUTLOOK}

There are several lines of investigation that need to be pursued in order to make substantial progress in low-energy nuclear physics.

The key issue here is how to deal rigorously with the consequences of QCD in the low energy (nonperturbative) realm. In the case of mesonic and single nucleon processes, methods based on the chiral symmetry of QCD offer perhaps the best way to addresss this challenge in the near term. Chiral perturbation theory is a reliable low energy procedure that is very successful in this regard. Nevertheless a number of important challenges remain:

i) In the realm of pseudoscalar meson interactions, we already have developed a very successful calculational scheme. The challenge here is to experimentally resolve some of the remaining thorny problems, such as the pion polarizability, and to extend the predictive power to higher energy. Present theoretical efforts which combine chiral effective theory with other techniques such as dispersion relations have been able to substantially increase the energy range over which solid predictions can be made.

ii) In the baryonic realm, it is essential not only to extend the predictive power to higher energy but also to solve the convergence issues which plague some observables. The same dispersive methods which are successful in the mesonic realm may be of utility here, as may well be new summations of the chiral series which respect the singularity structure of the basic amplitudes. Both approaches are being pursued at the present time.

iii) Dyson-Schwinger as well as other techniques, such as quenched lattice-QCD, have been used in order to compare theoretical and empirical values of the low energy 
constants. Such work will no doubt continue, and represents the ultimate challenge to any nonperturbative theoretical program.

In the case of multi-nucleon EFT:

i) A consistent and convergent power counting must be established for systems of nucleons and pions below the chiral symmetry breaking scale. This will allow for the calculation of processes that are both experimentally accessible and inaccessible; high precision calculations will be performed to make critical comparisons with low- and moderate-energy nuclear experiments. Such a comparison is vital to test the convergence of the theory for a variety of processes, but will also allow for the determination of nucleon properties. A good example of this would be the extraction of the nucleon anapole moment from low-energy electron scattering. Also, precise predictions of a multi-nucleon EFT will provide a bench mark for lattice-QCD calculations in much the same way as chiral perturbation theory presently does in the meson sector.

ii) Including electroweak gauge fields into systems with three or more nucleons is a short term priority. There are large theoretical uncertainties in potential model calculations of some electromagnetic processes of great importance in astrophysical environments. It is essential that these uncertainties be significantly reduced. Recall that BBN calculations and interpretation of SNO data are significantly impacted by uncertainties in theoretical nuclear physics predictions. Given the success in the two-nucleon sector, extension to the three-nucleon sector is important.

iii) The early efforts to implement an EFT description of multi-nucleon systems must continue, and be extended to nuclei of moderate atomic number. The calculations of the Argonne group will provide a bench mark. The systematic inclusion of chiral symmetry will allow for inelastic processes involving pions to be computed in the same framework as the computation of nuclear energy levels.

iv) As numerical studies of lattice-QCD will be unable to directly compute observables in multi-nucleon systems in the foreseeable future, in order for such efforts to have implications that are not purely academic, a partially-quenched multi-nucleon EFT is required.

v) High precision calculations continue to be carried out with $\operatorname{EFT}(\hbar)$. These calculations of low-energy processes can be continued to even higher orders, allowing for calculations with uncertainties at the fraction of $1 \%$-level. In conjunction with low-energy 
experiments of comparable precision, fundamental properties of the nucleon can be determined, in addition to allowing for precise predictions for processes of astrophysical interest.

vi) Finally, ultimately it must be shown that many-body nuclear physics methods emerge as a leading order effect in EFT. This will open the way for systematic improvement of these methods.

\section{COMPARISON OF U.S. AND GLOBAL EFFORT}

The development of EFT for particle and nuclear physics is a global effort. Important groups which focus on chiral perturbative studies - both in particle and nuclear physicsexist in England, Germany, Switzerland, Austria, Italy, France, Sweden, Canada, and in the United States. Groups which focus on multi-nucleon systems exist in France, Korea, Germany, Switzerland, England and the United States. In the latter case, the simultaneous need for knowledge of nuclear phenomenology and of quantum field theory techniques significantly restricts the number of physicists who have contributed in any meaningful way to the development of this area. It is only during the last few years or so that there has been substantial effort and progress in this field. Nevertheless, this progress has been impressive.

A good indication of the recent effort in chiral perturbation theory can be found in the proceedings of the recent Chiral Dynamics meetings which have taken place at MIT (1994) [23], at Mainz (1997) [24], and at JLab (2000) [25]. In each case about 100 physicists, both theorists and experimentalists got together to discuss developments in the field. Discussions were lively and a program setting out future work was developed. There was a good mix of $(\sim 70)$ senior and $(\sim 30)$ younger colleagues. However, the opportunities for tenure track positions has been quite limited.

An indication of the effort in the area of two-nucleon EFT can be found in the attendance at the second conference in this area during February of 1999 (proceedings from the first and second conferences on Nuclear Physics with Effective Field Theory can be found in Ref. [22]). Those in attendance at that meeting included twenty (20) active physicists in tenured or tenure track positions, and twenty (20) active physicists in non-tenure track positions (either postdoctoral fellows, students or research assistant professors) in the United States 凹. Since

\footnotetext{
1 The participant list can be found at the back of Ref. 22].
} 
the meeting, three (3) of the latter category have assumed tenure track positions, while the remaining seventeen (17) are waiting for permanent positions to become available to them.

\section{OTHER ISSUES}

With the large number of talented young physicists attracted to this area of research it is important for nuclear physics that tenure track positions (or their equivalent) be created in the near future. The vitality of the field (and of nuclear physics in general) depends upon the creativity and energy of young physicists. With the recent exceptions of the Jefferson Laboratory and RIKEN-BNL positions, the nuclear theory community has had only limited success in recruiting such talent into its ranks and therefore a serious effort must begin now. The alternative is intellectual sterility.

Given that EFT has long been the lingua franca of the many branches of particle physics, the flow of information and understanding has largely been into nuclear physics. However, recently, the expertise that we have developed in systems with large scattering lengths (i.e. nuclear physics) has been applied to the physics of BEC with great success. It is clear that with BEC becoming only recently accessible to experimental investigation, the overlap between the EFT program in nuclear physics and condensed matter physics will continue to grow. During the recent EFT program at the Institute for Nuclear Theory in Seattle, Washington, it became clear that the EFT tools being developed for high precision atomic calculations will be influenced and will influence those being developed for nuclear systems. (Incidentally, this meeting was oversubscribed by a factor of two.)

It is also the case that realistic application of lattice-QCD results to experimental physics requires the use of EFT methods in order to extrapolate from the region of calculability into the regime chosen by nature. In addition, the confrontation of lattice-QCD (or other theories) calculations with empirical findings at low energy is most efficiently carried out by comparing calculated and measured low energy constants of an EFT rather than by dealing with matters on a process by process basis.

Finally, it is of the utmost importance that the nuclear physics community maintain facilities that will allow for high precision, low-energy measurements of observables in fewnucleon systems. 


\section{ACKNOWLEDGEMENTS}

This work is supported in part by the U.S. Dept. of Energy under Grants No. DEFG03-97ER4014 (MJS) and the National Science Foundation (BRH). 


\section{REFERENCES}

[1] See, e.g. D. Kaplan, Effective Field Theories, in Proc. 7th Summer School in Nuclear Physics, nucl-th/9506035; H. Georgi, Effective Field Theory, in Ann. Rev. Nucl Sci. 43, 209 (1995); A. Manohar, Effective Field Theories, in Schladming 1996: Perturbative and Nonperturbative Aspects of Quantum Field Theory, hep-ph/9606222.

[2] S. Weinberg, Phys. Rev. Lett. 18, 188 (1967); Phys. Rev. 166, 1568 (1968); Physica A96, 327 (1979).

[3] J. Gasser and H. Leutwyler, Ann. Phys. 158, 142 (1984); Nucl. Phys. B250, 465 (1985).

[4] U. -G. Meißner, Essay for the Festschrift in honor of Boris Ioffe, to appear in the 'Encyclopedia of Analytic QCD', edited by M. Shifman, to be published by World Scientific. hep-ph/0007092.

[5] S. Weinberg, Phys. Lett. B251, 288 (1990); Nucl. Phys. B363, 3 (1991); Phys. Lett. B295, 114 (1992).

[6] S. R. Beane, P. F. Bedaque, W. C. Haxton, D. R. Phillips and M. J. Savage, Essay for the Festschrift in honor of Boris Ioffe, to appear in the 'Encyclopedia of Analytic QCD', edited by M. Shifman, to be published by World Scientific. hep-ph/0008064.

[7] Particle Data Group, Phys. Rev. D54, 1 (1996).

[8] Yu. M. Antipov et al., Z. LPhys. C26, 495 (1985).

[9] Yu. M. Antipov et al., Phys. Lett. B121, 445 (1983).

[10] T.A. Aibergenov et al., Czech. J. Phys. 36, 948 (1986).

[11] D. Babusci et al., Phys. Lett. B277, 158 (1992).

[12] E. Jenkins and A. V. Manohar, Phys. Lett. B255, 558 (1991); Phys. Lett. B259, 353 (1991); Talks presented at the Workshop on Effective Field Theories of the Standard Model, Dobogoko (1991).

[13] See, e.g. T.R. Hemmert et al., Phys. Rev. D62, 014013 (2000).

[14] S. R. Sharpe and N. Shoresh, Phys. Rev. D62, 094503 (2000); hep-lat/0011089; M. Golterman, hep-ph/0011084.

[15] See, e.g. J. Gasser and M.E. Sainio, Eur. Phys. J. C6, 297 (1999).

[16] See, e.g., J.F. Donoghue and B.R. Holstein, Phys. Rev. D48, 137 (1993).

[17] See, e.g. J. Kambor, C. Wiesendanger, and D. Wyler, Nucl. Phys. B465, 215 (1996).

[18] See, e.g., R. Flores-Mendieta et al., Phys. Rev. D62, 034001 (2000).

[19] V. G. S. Nakamura, T. Sato and K. Kubodera, Private Communication.

[20] M. Butler and J.-W. Chen, Nucl. Phys. A675, 575 (2000); M. Butler, J.-W. Chen, and X. Kong, (2000), nucl-th/0008032.

[21] S. Ying, W. C. Haxton and E. M. Henley, Phys. Rev. D40, 101 (1989); Phys. Rev. C45, 1982 (1992). 
[22] Proceedings of the Joint Caltech/INT workshop on Nuclear Physics with Effective Field Theory, edited by R. Seki, U. van Kolck and M. J. Savage, published by World Scientific (1998), ISBN 981-02-3596-8; Proceedings of the INT workshop on Nuclear Physics with Effective Field Theory II, edited by P. F. Bedaque, M. J. Savage, R. Seki and U. van Kolck, published by World Scientific (1999), ISBN 981-02-4181-X.

[23] Chiral Dynamics: Theory and Experiment, ed. A.M. Bernstein and B.R. Holstein, Springer-Verlag, New York (1995).

[24] Chiral Dynamics: Theory and Experiment, ed. A.M. Bernstein, D. Drechsel, and T. Walcher, Springer-Verlag, New York (1998).

[25] Chiral Dynamics 2000, JLab, July 2000 (to be published). 